\title{
Human Computer Interaction During Clinical Decision Support With Electronic Health Records Improvement
}

\author{
Katerina V. Bolgova, ITMO University, Saint Petersburg, Russia \\ Sergey V. Kovalchuk, ITMO University, Saint Petersburg, Russia \\ (iD https://orcid.org/0000-0001-8828-4615 \\ Marina A. Balakhontceva, ITMO University, Saint Petersburg, Russia \\ Nadezhda E. Zvartau, National Almazov Medical Research Centre, Saint Petersburg, Russia \\ Oleg G. Metsker, ITMO University, Saint Petersburg, Russia
}

\begin{abstract}
This study investigated the most common challenges of human-computer interaction (HCI) while using electronic health records (EHR) based on the experience of a large Russian medical research center. The article presents the results of testing DSS implemented in the mode of an additional interface with the EHR. The percentage of erroneous data for two groups of users (with and without notifications) is presented for the entire period of the experiment and the weekly dynamics of changes. The implementation of CDSS in the supplemented interface mode of the main medical information system (MIS) has had a positive effect in reducing user errors in the data. The results of users' survey are presented, showing a satisfactory evaluation of the implemented system. This study is part of a larger project to develop complex CDSS on cardiovascular disorders for medical research centers.
\end{abstract}

\section{KEYWORDS}

Clinical Decision Support Systems, Electronic Health Records, Healthcare Quality, Human-Computer Interaction, Medical Data Analysis

\section{INTRODUCTION}

In recent years, so-called personalized medicine has become widespread and developed (Hamburg \& Collins, 2010). The transition to personalized medicine within the paradigm of P4 medicine (Predictive, Preventive, Participatory and Personalized) (Sobradillo, Pozo, \& Agustí, 2011) is inseparably linked to the transition from evidence-based (or volume-based) medicine to value-based approach. This approach can be expressed as the ratio of the change in the quality of patient life and the number of resources spent on the treatment (the number of tests, procedures, prescription drugs, medical hours, etc.) (Bae, 2015). The most valuable is the care delivery, which is based on rigorous scientific knowledge and has the minimum cost with maximum benefit for patients. Costs are determined not so much by the 
money spending of treatment, as by the time and effort the patient spends on the treatment, and also by the number of staff-hours. That is why it is necessary to carry out comprehensive efforts that will reduce costs and improve the quality of treatment to provide a quality healthcare delivery.

One of such areas is the improvement of human-computer interaction (HCI) between physicians and medical information systems. On the one hand, satisfaction with the system will allow physicians to enter more correctly and quickly all required information, and on the other hand, it will improve the quality of the data itself for their subsequent analysis.

The Western world invests significant resources to digitize healthcare with a particular emphasis on the creation of an integrated electronic health record (EHR) to improve the efficiency and quality of care (Fitzpatrick \& Ellingsen, 2013). EHR offers several critical advantages over paper health records (PHR) related to the quality of care, efficiency and high level of patient safety (Hsiao, Hing, \& Ashman, 2014). In addition, EHR is a valuable source of quality assurance of medical practice and research (Middleton, 2014). Practical use of EHR requires structured data entry. It can be a challenge for users due to EHR method of interaction, which does not coincide with their mental models and do not meet the requirements of document flow (Belden, Grayson, \& Barnes, 2009; Friedberg et al., 2013). Poorly designed and cumbersome user interfaces of EHR input data can complicate the structured data-entry that will lead to a deterioration of data quality and incompleteness of data (Khajouei, Peek, Wierenga, Kersten, \& Jaspers, 2010). Consequently, this can lead to suboptimal functioning of information systems of medical technology, integrated into the EHR, for example, computerized support for making clinical decisions (CDSS). CDSS is one of the most effective strategies for improving clinical decisions (Roshanov et al., 2013). CDSS often requires a large amount of data about the patient (demographic data, data on complaints, symptoms, medical history, physical examination, laboratory and other tests).

Despite the fact that researchers aim to improve the quality of service, most of them reported only about the improvement of the professional performance and attempted to identify the critical success factors for CDSS have provided conflicting results (Bright et al., 2012; Roshanov et al., 2013). CDSS take their information from forms were filled in EHR and can provide incomplete advice due to incomplete and unstructured EHR data (Jaspers, Smeulers, Vermeulen, \& Peute, 2011). However, often the application of the existing approaches to design DSS health care and medicine is faced with significant difficulties for several reasons considered further concerning hospital practice. First, health information systems (HIS) in use often do not provide the functionality of DSS or the possibility to add such options. DSS deployment with existing HIS will complicate physicians' work because with filling paper records and entering data into HIS they will have to double the data in the CDSS.

Meanwhile, as mentioned above, improvement of human-computer interaction in EHR demands not only technical solutions but also facilitation of physicians' understanding of the importance of such systems for their routine practice and further use of data stored in such systems.

The rest of the paper is organized as follows. In section 2 related works are presented. In section 3 we describe several problems connected with bad organized human-computer interaction in medical information systems (MIS), and also, we propose the way to solve some of these issues by introducing clinical decision support system developed as an extension of MIS. Section 4 describes the goals of experiments, mistakes that we find in EHR, and the scheme of extension for MIS is proposed. In section 5 results of experimental sample exploitation of triggers for MIS are described. Finally, Sections 6 and 7 present discussions and conclusions.

\section{RELATED WORKS}

HCI are frequently associated with systems' usability and user satisfaction. Usability is a widely recognized feature of designing industrial systems and products, consumer software and other. The requirements for usability in these areas are not limited only by the user-friendly interfaces. In general, the application of HCI methods must meet the design's requirements for the development of 
user-centered, participatory and interactive systems. The main aspects of HCI are (i) methodologies and interface design, (ii) methods for implementing interfaces and their evaluation, (iii) interaction methods and descriptive and predictive models. CDSS links clinical observations with medical knowledge and experience and thus improves the quality of decisions and medical care provided.

The central attempts in the development of CDSS have been aimed at complex support of most aspects of clinical problems. However, this kind of system has become widespread mainly in the field of pharmacies, billing and telemedicine (Curtain \& Peterson, 2014; Mohktar, Lin, Redmond, Basilakis, \& Lovell, 2013). Partly this is because the developed systems do not meet all the usability requirements mentioned above. In contrast, the use of the principles of usability in the development of EHR and CDSS is sporadic and unsystematic, in part because of a lack of attention and effective design and evaluation structures. The authors (Zhang \& Walji, 2011) define HCI as a study of the interactions of people with computers, and how to create computer systems that are easy, fast and productive to use for people.

Another group of researchers emphasizes the importance of the improved HCI paradigm for the presentation of recommendations. According to (Sittig et al., 2008) one of the problems with the ease CDSS using is to make them work unobtrusively in the background, but still be useful and specific. CDSS should remind users of things that they may have forgotten, misinterpreted or missed before deciding instead of correcting users after an error occurred. Currently, a serious problem is the huge number of pop-up notifications. When patients are subjected to frequent and overwhelming warnings in daily practice, clinicians may become insensitive to warnings and therefore may pay less attention or even redefine them without offering any significant reasons.

There are also a few studies that compare different theories of HCI applied for healthcare. The authors (Wiser, Durst, \& Wickramasinghe, 2018) conclude that the Activity Theory is most suitable for the tasks of describing such complex systems like MIS, fixing changes and evaluate of the impact of these changes on all actors.

This phenomenon is called "alert fatigue" (Rose \& Joshi, 2018), and it reflects how busy clinicians become tolerant to safety warnings. This symptom can be extremely dangerous, as the critical alerts, warning of impending or severe harm to the patient, cannot be heard with the annoying warnings or clinically meaningless. Potential solutions to reduce the effect of "alert fatigue" on decisions makers are (1) increasing alert specificity; (2) tier alerts according to severity; (3) apply human factors principles when designing alerts, and include only high-level (severe) alerts in an alert set; (4) tailor alerts to patient characteristics; (5) customize alerts for physicians (Marcilly, R., Ammenwerth, E., Roehrer, E., Niès, J., \& Beuscart-Zéphir, 2018).

Curiously enough, computer warnings aimed at improving safety can lead to an increased probability of patient's damage. Since EHR systems are widely used in the modern health environment, fatigue is recognized as a serious, unintended consequence, as well as a severe problem of patient safety in case of drug-drug interaction alerting (Slight et al., 2013). The research (Payne et al., 2015 ) is aimed at establishing preferred strategies for submitting drug-drug interaction (DDI) alerts, recommendations have been generated for interfaces, including the use of terminology, visual signals, minimal text, formatting, content and reporting standards for ease of use. All clinicians involved in the use of drugs should be able to view warnings about DDI by other physicians. The redefinition levels are typical but cannot be a good measure of efficiency. Warnings about DDI in clinical decision support require significant improvements.

CDSS usability issues are associated with both clinical and technical problems. (Sittig et al., 2008) argue that it is necessary to begin initial efforts to develop more reliable methods for identifying, describing, evaluating, collecting, cataloging, synthesizing and disseminating best practices for the development, implementation, maintenance, and evaluation of clinical solutions. Previously, many CDSS were not well integrated with the CPOE computer-based physician order entry (CPOE), and physicians preferred to ignore CDSS only because of the requirement of "double data entry" that interrupts patient care. As more and more CDSS is integrated into EHR systems, the problem of dual 
data entry is no longer a major problem for clinicians, although several decision support systems for diagnosis remain autonomous rather than integrated into EHR systems (Miller et al., 2015).

\section{PROBLEM DEFINITION}

Basically, EHR represents the case history of a patient and contains information about patient complaints, investigations, and treatment obtained during visits to the medical center. The information includes general information about the patient (age, gender, etc.), results of medical examination by a doctor (diagnosis and prescriptions) and data about various medical procedures (medical tests results, surgery). The incomplete case history may result in an underestimation of the severity of the disease or adverse drug interactions, jeopardize the integrity of the information, and lead to mistakes and misdiagnosis, thereby interfering with patient safety or decreasing the quality of health care. In the current study, we focused on the analysis of information filled by the treating physician during outpatient visits.

Within our study, we consider the model-based generalized architecture of clinical decision support system based (CDSS) developed as an extension of medical information system (MIS) for various kind of decision makers and experts (Figure 1). The proposed enhanced architecture may be considered as a generalized view on the implementation ways of previously proposed model-based CDSS concepts (Krikunov et al., 2016; Syomov et al., 2016). The architecture includes three main scales to consider data and model processing as well as key users interaction. Also, the architecture is divided into three main sub-systems:

- Basic MIS modules (client- and server-side) considered as existing within a hospital infrastructure;

- Server-side DSS modules responsible for centralized management of data, knowledge, and client interaction;

Figure 1. Generalized architecture of CDSS

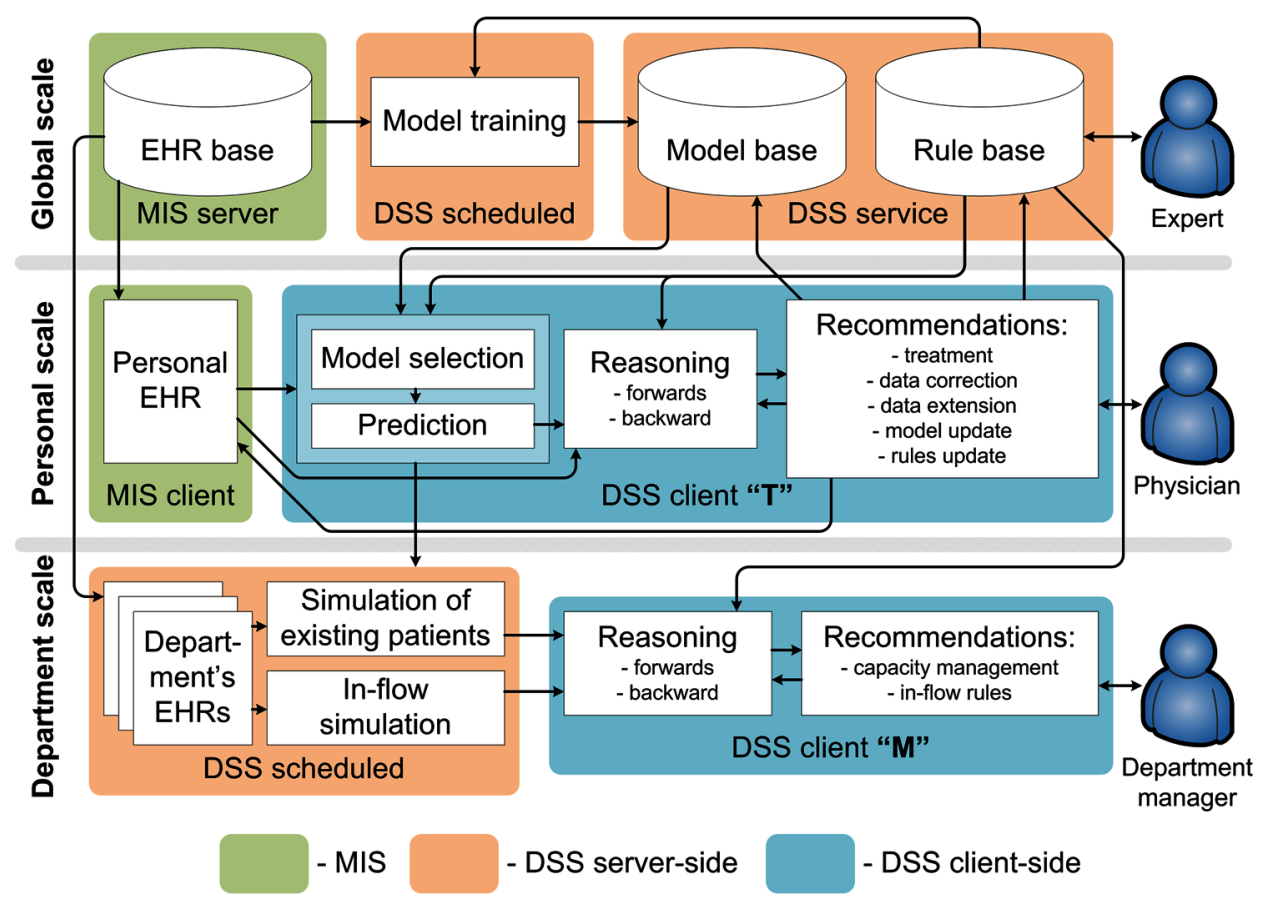


- Client-side DSS modules responsible for local human-computer interaction to support certain activities of a user.

Global scale includes basic data (EHR is considered as the main data source in our studies), knowledge (we use rule base as a simple representation of static knowledge) and models (which may also be considered as knowledge) in corresponding bases. Additionally, this scale includes processes of knowledge control with automatic or semi-automatic model training and knowledge expression and management performed by an expert. Personal scale represents the key use case of the CDSS where support of physician's decisions is provided. Here basic personalized EHR-based representation in MIS is extended with predictive (mainly data-driven) models which extend available facts. Knowledge-Based reasoning with EHR and predicted facts is used to infer a set of recommendation for a) physician activity; b) support end extension of knowledge and model bases; c) requesting additional data. Finally, department scale represents support for managerial tasks usually solved by heads of hospitals or departments. In particular, this scale includes predictive (both data-driven and simulation) models to analyze inflow and processing of patients, a load of a hospital or a department and support of strategic decision making for reaching of the higher performance of the organization.

The proposed architecture may be implemented in various ways. Still, an important part in the approach is interaction with key users (experts, physicians, managers) during support and using of CDSS. Within the current study, we were focused on the investigation of human-computer interaction in CDSS with special attention to controlling and improvement of EHR. Further sections describe the implementation of the proposed generalized architecture and experimental study in a hospital during providing care for outpatients with arterial hypertension.

\section{Experimental Setting Details}

This section describes experimental settings, key features influencing the study, as well as implemented CDSS. Within the study, we considered a process of providing care for outpatients with arterial hypertension (AH). Disease development and treatment process in $\mathrm{AH}$ cases is often complicated by various comorbidities and external factors (habits, physical activity, mental health, etc.). Such complexity may cause potential mistakes, inconsistencies, missing and erroneous data in EHR. Therefore, AH was selected as a target disease within the research. Revealed mistakes in EHR with AH patients were systematically arranged into six classes. The rule base of the developed CDSS was extended with corresponding rules to prevent and correct the mistakes during out-patient visit. This section presents the details on analysis of AH patients and EHR, inferred mistake classes and development of CDSS.

\section{Case Study}

$\mathrm{AH}$ as a widely spread example of chronic cardiovascular disease, which may lead to severe health conditions like acute myocardium infarction (AMI) or stroke. AH is a multifactorial disease, and its contraction causes are usually unknown. The manifestation, progression, and treatment outcomes of $\mathrm{AH}$ are associated with various factors, such as the population genetic trait, comorbidities, climate, and other risk factors. In this regard, current hypertension guidelines provide for a large number of treatment options without apparent differences in the treatment effectiveness.

Federal Almazov North-West Medical Research (Almazov Centre) as one of the leading cardiological centers in Russia provide extended care and consulting for outpatients with AH. From 2010 to 2016 Almazov center provide treatment to 44393 patients with chronic diseases associated with AH. Also, the MIS database contains EHR of treatment episodes (3252) these patient diseases of the heart circulatory system (angina, ACS, myocardial infarction). At the same time, EHRs are laboratory test data (cholesterol level, glucose level, general blood test, etc.), instrumental tests (electrocardiography, echocardiography, etc.), comorbidities (diabetes, atherosclerosis, dyslipidemia, fibrillation, COPD, CKD), observation data (BMI, age, gender), event logs (activity, date, time, 
Department, responsible person), anamnesis and other records. In General, the group of patients does not differ significantly in characteristics. Of the total number of patients, $37 \%$ are men and $63 \%$ are women. At the same time, the average age of men is 52 years, and the average age of women is 60 years. Obesity (BMI > 30) is diagnosed in $27 \%$ of men and $33 \%$ of women. Elevated (>4.9) cholesterol in $81 \%$ of patients for whom laboratory studies of lipids were carried out. The increased blood glucose level ( $>5$ ) was diagnosed in $84 \%$ of patients.

Observed diversity of the Almazov Centre's patients enables enhanced analysis of the AH patient population in Russia. For example, Figure 2 shows selected results in the analysis of AH patients in Almazov Centre. Figure 2a shows clustering of episodes (using proximity complex measure) with selected popular chronic diseases: AH (green), diabetes mellitus (blue), and chronic heart failure (red). The analysis shows a) existence of various clusters of AH patients showing diverse condition groups and treatment patterns applied; b) presence of AH patients inconsistent clusters (see clusters for diabetes mellitus); c) widely spread combination of $\mathrm{AH}$ with other chronic diseases (also with heart failure, which has highly diverse patterns, so it doesn't form consistent clusters). In addition, dynamics analysis (Figure 2b) shows a constantly growing portion of elderly people in AH population. Although this could be considered as good evidence (people live longer), the complexity of that cases significantly grows. The analysis lets to predict high (and growing) complexity of $\mathrm{AH}$ cases and more complex and uncertain care process. Therefore, we selected $\mathrm{AH}$ as a target disease within our experimental study in CDSS and EHR improvement after HCI support in clinical practice.

\section{Mistakes in EHR of AH Patients}

Since the original system was not designed for data analysis and carried out more administrative function, the quality of the data does not confirm the entry requirements for analysis. As we described earlier (Bologva, Prokusheva, Krikunov, Zvartau, \& Kovalchuk, 2016), we selected six classes of the most common mistakes in EHR to be analyzed. Total count of analyzed records was 32158.

Mistakes' classes and frequency of their occurrence were the following:

Figure 2. AH patients observed in Almazov Centre: (a) Clustering of episodes with selected chronic diseases and AH; (b) Six-year trends in patients with severe hypertension in different age groups

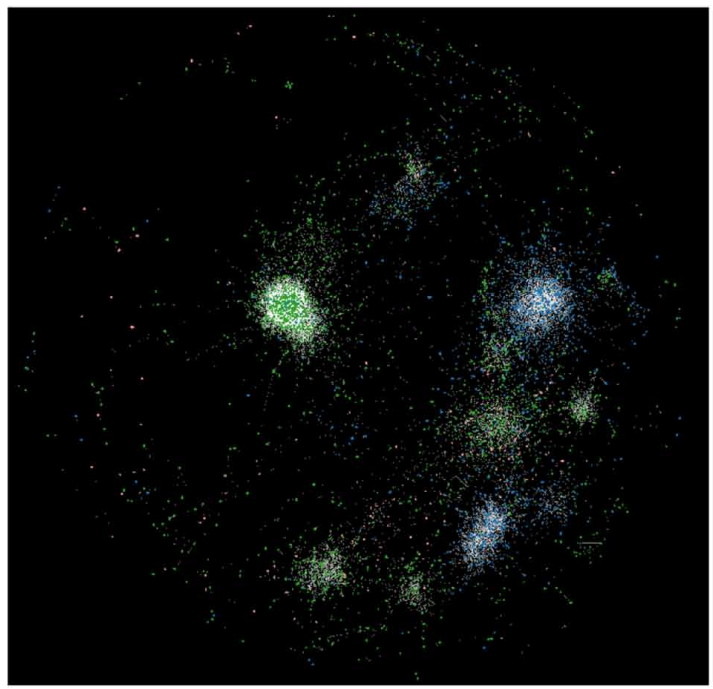

(a)

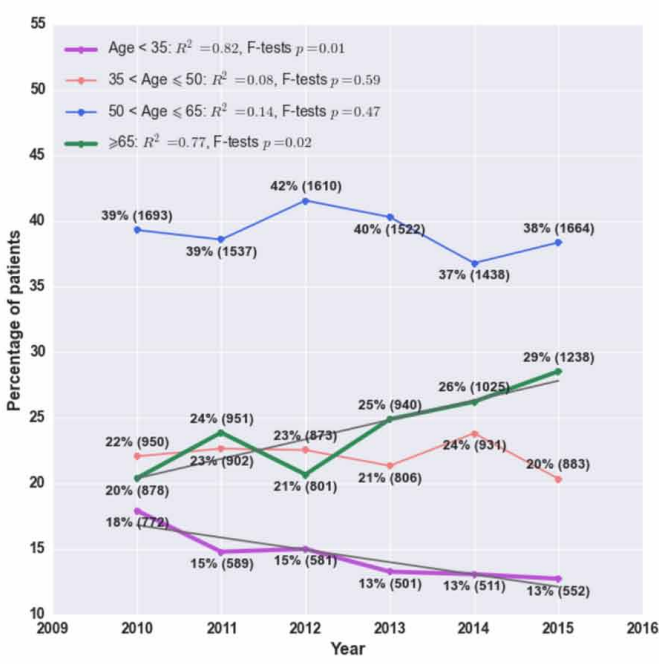

(b) 
1. Mistakes in drug prescriptions: (a) typos and (b) brand drug name was written before the international nonproprietary name (212361 [57\%] of 369417 total prescript drugs, and 31598 [12\%] of prescription cases of 261815 , respectively);

2. No blood pressure data (3283 [11\%] records);

3. No BMI data (26130 [81\%] records);

4. Diagnosis was not properly structured: it was difficult to retrieve information about the underlying disease, concomitant disease, and complications (20902 [65\%] records);

5. We assume erroneous situation if (a) attached laboratory results of lipids profile were above threshold levels; (b) doctor prescribed statins and (c) term "dyslipidemia" was not in the field 'diagnosis' (163 [0.5\%] records);

6. Multiple recording methods of the same information. For example, frequent use of both "-" and "not complicated" in the field 'concomitant diseases' (16500 [51\%] and 3887 [12\%]) records respectively).

These kinds of mistakes make records unsuitable for analysis, and we can lose valuable information or medical cases. We suggested ill-designed HCI is the leading cause of such mistakes.

\section{CDSS Implementation}

Previous studies determine the results of the current experiment. In particular, papers (Bologva et al., 2016; Semakova, Zvartau, Bolgova, \& Konradi, 2016) describe the analysis of data from MIS for the most common mistakes, and it was suggested that the developing of a CDSS that integrates with an existing MIS could solve the problem. Authors of the paper (Syomov et al., 2016) provide a general approach that was taken as the basis for the introduction of such a CDSS. As a result, an experimental sample of an integrated CDSS with a limited functional was developed and implemented for the current experiment (the CDSS architecture is shown in Figure 3). The functionality of CDSS was intended to be limited for fixing errors and notifying users of a particular error. This was done to focus on the effect in work and the perception of users from the operation of such a system.

Two EHR subsection were allocated for the experiment:

1. Subsection "General inspection", in which the fields are distinguished: height, weight, waist circumference (WCirc), heart rate (HR), arterial blood pressure (ABP). We choose this fields to control because they are important for AH patients. For this section, two error classes are considered: miss - if the field is left blank and error - if the field contains erroneous data (for example, letters in a numeric field, or the value is out of bounds);

Figure 3. The architecture of integrated CDSS

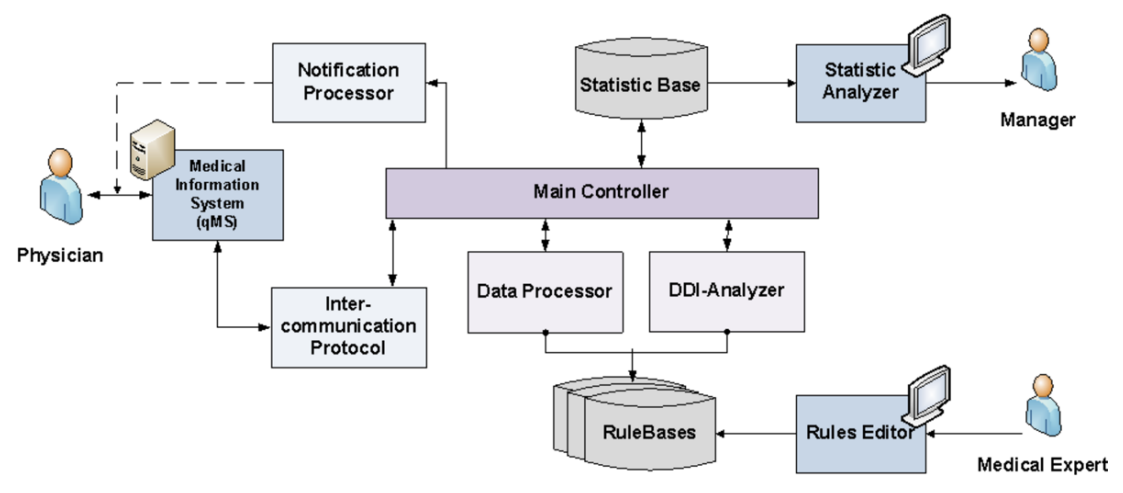


2. Subsection "Recommendations", which verifies the compatibility of prescription drugs. For this purpose, we create a knowledge base of drugs that are often prescribed for patients with AH. Every drug in this base contains information about harmful or negative interaction with other drugs. If in the prescription there is at least one negative drug-drug interaction a warning will be issued.

\section{EXPERIMENT RESULTS}

The testing period of the experimental sample of the CDSS was ten full weeks. In the testing involved seven physicians: men and women 30-45 years old which could be characterized as users with shallow computer skills. They were divided into two groups: 4 doctors worked with included mistakes notifications (with triggers), and 3 of them in the "fixing actions" mode, that is, notifications were not shown (without triggers). The results are shown in Table 1.

Statistics for specific mistakes are presented in Table 2. Special attention was paid to the "General inspection" since the vast majority of errors was previously associated with either the lack of data in specific fields or the erroneous writing of them. The table shows the percentage of missing or erroneous data that was recorded in the system and the percentage of missing or erroneous data that remained in the system after the form was closed.

In Figure 4, the results from Table 2 are presented graphically. The graphs show that the number of omissions and erroneous data remaining in the MIS has decreased noticeably in the focus group that works with enabled notifications (with triggers). Figure 5 shows the percentage of the initial omissions or user errors recorded in the system for each of the ten weeks.

In addition to analyzing the results of mistakes, a survey was carried out by physicians who worked with the system with enabled notifications. The purpose of the survey was to find out how the system is convenient and useful in the work of a doctor. The results of the survey showed that most physicians were able to adapt to the new mode of operation, all noted that notifications were useful to them in their work. However, almost all respondents noted that it was not more convenient for the system to work. This is since there are additional pop-up windows and the adaptation to them requires a slightly longer time interval. Also, for now, we cannot overcome the «alert fatigue» phenomenon to the full extent. However, we will discuss with doctors what factors affect fatigue, what hours are the most stressful and together with doctors we will develop some scenarios to solve this problem.

Table 1. General results of system testing

\begin{tabular}{|l|l|l|}
\hline \multicolumn{2}{|c|}{ With Triggers (\%) } & Without Triggers (\%) \\
\hline Subsection “General inspection” & \multicolumn{2}{|l|}{} \\
\hline At least with one mistake & $49,1 *$ & 63,7 \\
\hline Remained wrong & 8,7 & 42,2 \\
\hline Subsection “Recommendations” & $25,5 * *$ & $24 * * *$ \\
\hline Recorded messages about drugs incompatibility & 7 & 0 \\
\hline Information changed after the message & 7 & 0 \\
\hline
\end{tabular}

${ }^{*} 84.7 \%$ of mistakes were committed in the first weeks.

** Some of the reports were about "Mutual intensification of the hypotensive effect" (64.3\%) and "Reduction of the effect of one drug in interaction with another" $(21.4 \%)$

${ }^{* * *}$ Some of the reports were about "Mutual intensification of the hypotensive effect" $(37,5 \%)$ and "Reduction of the effect of one drug in interaction with another" $(12,5 \%)$ 
Table 2. Statistics for specific mistakes

\begin{tabular}{|c|c|c|c|}
\hline & & With Triggers (\%) & Without Triggers (\%) \\
\hline \multirow{2}{*}{ Height } & Missing & 7,3 & 25,5 \\
\hline & Remained wrong & 2,9 & 23,5 \\
\hline \multirow{2}{*}{ Weight } & Missing & 24,6 & 27,5 \\
\hline & Remained wrong & 8,7 & 21,6 \\
\hline \multirow{2}{*}{ WCirc } & Missing & 20,3 & 49,1 \\
\hline & Remained wrong & 7,25 & 41,2 \\
\hline \multirow{2}{*}{ HR } & Missing & 23,2 & 39,2 \\
\hline & Remained wrong & 10,14 & 29,5 \\
\hline \multirow{2}{*}{$\mathrm{ABP}$} & Missing & 13 & 15,7 \\
\hline & Remained wrong & 1,5 & 11,7 \\
\hline \multirow{2}{*}{ Height } & Error & 1,5 & 0 \\
\hline & Remained wrong & 0 & 0 \\
\hline \multirow{2}{*}{ Weight } & Error & 7,25 & 9,8 \\
\hline & Remained wrong & 1,5 & 9,8 \\
\hline \multirow{2}{*}{ WCirc } & Error & 0 & 0 \\
\hline & Remained wrong & 0 & 0 \\
\hline \multirow{2}{*}{ HR } & Error & 8,7 & 5,9 \\
\hline & Remained wrong & 0 & 5,9 \\
\hline \multirow{2}{*}{$\mathrm{ABP}$} & Error & 18,8 & 30,3 \\
\hline & Remained wrong & 4,35 & 21,2 \\
\hline
\end{tabular}

Figure 4. The percentage of user mistakes during the system testing period: (a) The total number of recorded mistakes; (b) The number of mistakes remaining in the MIS (after corrections)
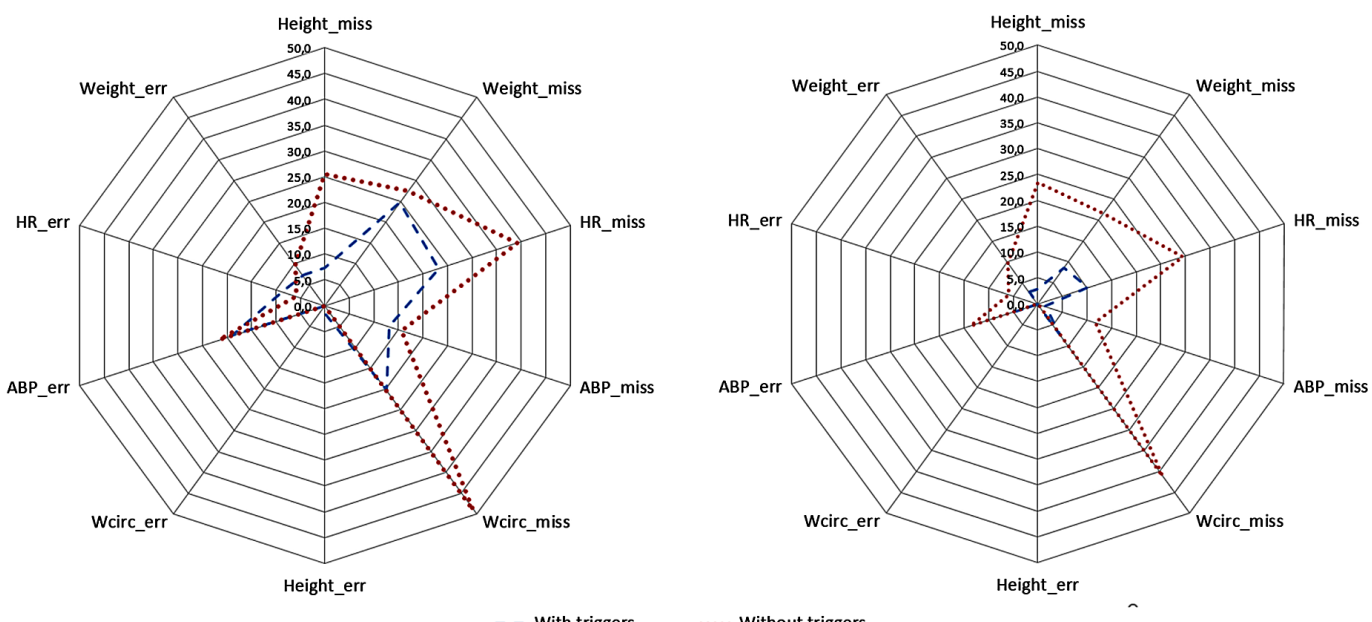
Figure 5. The percentage of user mistakes by week: (a) The focus group with enabled notifications; (b) Focus group with notifications turned off

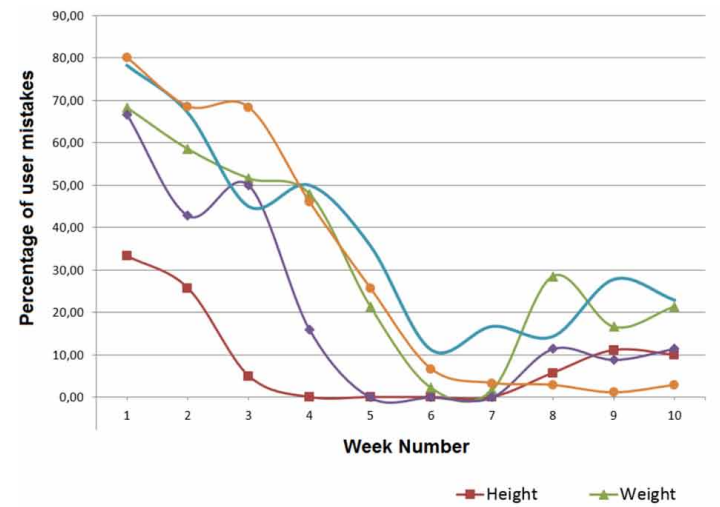

(a)

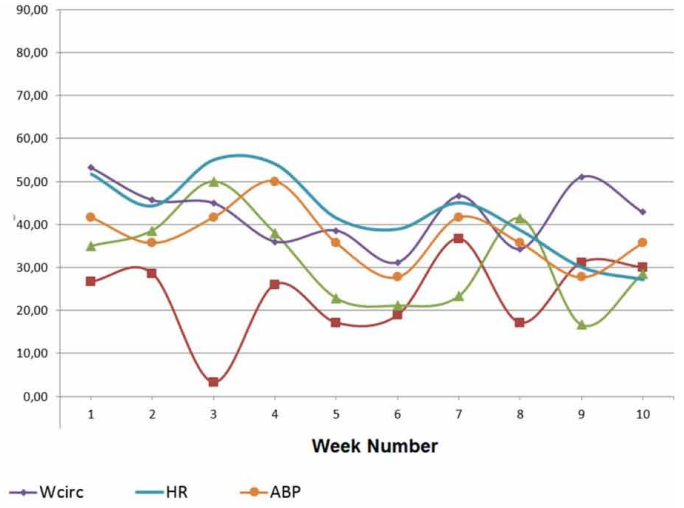

(b)

\section{DISCUSSION}

In Figure 5 we see the curves noticeably decrease, and then increase slightly, but at the moment it is difficult to predict their behavior in the future, so additional testing is necessary. Figure 4 shows the average mistakes value (solid blue line). As mentioned in the previous work (Bologva et al., 2016), we use the DMAIC cycle (George, 2010) to develop the system, and we have already passed one turn, then we need to clarify the recommendations of users, make some changes to the system and conduct further testing. We assume that without changes the behavior of the curve will not change much later (Figure 6, the blue dashed line). However, when following the DMIAC cycle and considering the interests and requirements of users, the behavior of the curve can be a similar (Figure 6 green line). Thus, we need to achieve the behavior shown in Figure 6. That in its turn, when approximated, will give a curve very similar to the Fogg curve (Fogg \& BJ, 1998). And if we can get this result, we can use the Fogg model to predict behavior and to control behavior through motivation, training, and triggers.

Figure 6. The average mistakes value and the further behavior

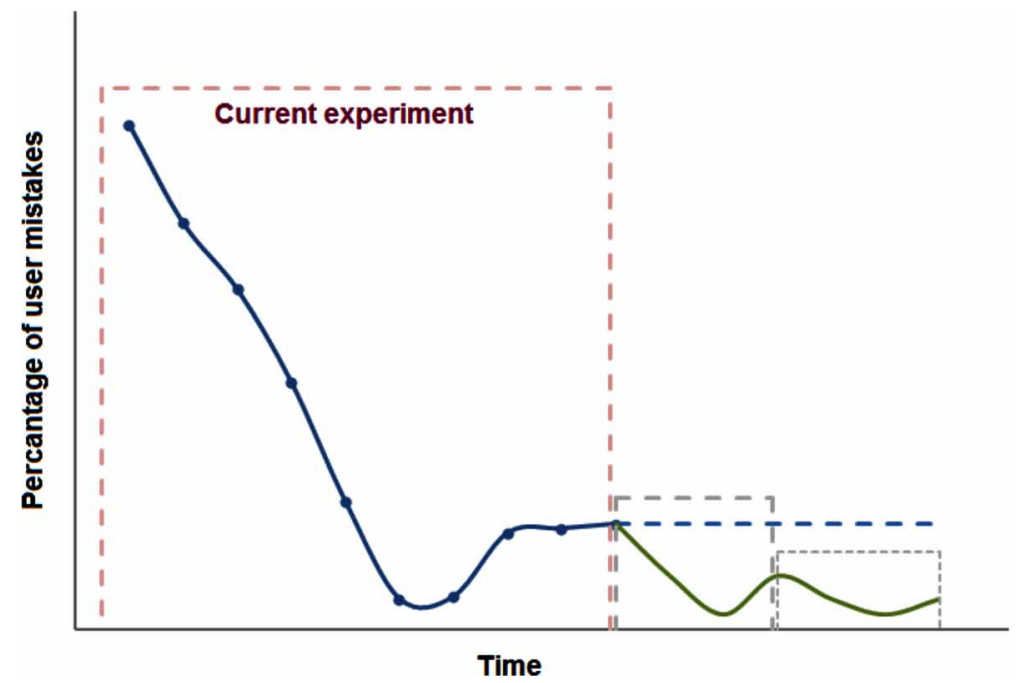


An important issue to be considered is the integration of the proposed approaches in a framework of automatic learning of CDSS to support the activity of users from various groups. We can treat this task as reinforcement learning problem (Sutton \& Barto, 1998). In that case models and knowledge bases in the proposed generalized architecture may be controlled by an intelligent algorithm which may constantly improve the HCI process considering a) issues of the particular hospital; b) personalized behavioral patterns of the users; c) changing in behavior and knowledge under control of the CDSS. This leads to the idea of symbiosis of users and information system evolving towards a better level of service providing.

\section{CONCLUSION AND FUTURE WORK}

The proposed idea of implementation of CDSS in the supplemented interface mode of the main MIS has had a positive effect in reducing user errors in the data (EHR in the considered case). We observe shifts towards better data quality after introducing controllable HCI having decreased in EHR mistakes as a goal. Furtherly we consider the development of more advanced, flexible, and transparent techniques in HCI to make the next iteration in MIS-based solution improvement. Future research directions include enrichment of knowledge and model bases with new entities, an extension of the proposed solution for HCI with various classes of users, application of the solution in various disease treatment (including out- and in-patients, consulting, etc.), and others.

We considered the approach to improve information (EHR in our case) through explicit control of $\mathrm{HCI}$ as a promising research direction where the close collaboration of human and machines can reach a new level. The idea may be applied widely beyond the area of CDSS in multiple information systems where HCI plays a crucial role in the quality of the process or service.

\section{ACKNOWLEDGMENT}

The research was supported by the Russian Science Foundation (project No. 17-15-01177). 


\section{REFERENCES}

Bae, J. (2015). Value-based medicine: Concepts and application. Epidemiology and Health, 37, 1-5. doi:10.4178/ epih/e2015014 PMID:25773441

Belden, J. L., Grayson, R., \& Barnes, J. (2009). Defining and Testing EMR Usability: Principles and Proposed Methods of EMR Usability Evaluation and Rating. UM System. Retrieved from https://mospace.umsystem.edu/ xmlui/handle/10355/3719

Bologva, E. V., Prokusheva, D. I., Krikunov, A. V., Zvartau, N. E., \& Kovalchuk, S. V. (2016). Human-Computer Interaction in Electronic Medical Records: From the Perspectives of Physicians and Data Scientists. Procedia Computer Science, 100, 915-920. doi:10.1016/j.procs.2016.09.248

Bright, T. J., Wong, A., Dhurjati, R., Bristow, E., Bastian, L., Coeytaux, R. R., \& Lobach, D. et al. (2012). Effect of clinical decision-support systems: A systematic review. Annals of Internal Medicine, 157(1), $29-43$. doi:10.7326/0003-4819-157-1-201207030-00450 PMID:22751758

Curtain, C., \& Peterson, G. M. (2014). Review of computerized clinical decision support in community pharmacy. Journal of Clinical Pharmacy and Therapeutics, 39(4), 343-348. doi:10.1111/jcpt.12168 PMID:24806361

Fitzpatrick, G., \& Ellingsen, G. (2013). A Review of 25 Years of CSCW Research in Healthcare: Contributions, Challenges and Future Agendas. Computer Supported Cooperative Work, 22(4-6), 609-665. doi:10.1007/ s10606-012-9168-0

Fogg, B., \& BJ. (1998). Persuasive computers. In Proceedings of the SIGCHI conference on Human factors in computing systems - CHI '98 (pp. 225-232). New York: ACM Press. doi:10.1145/274644.274677

Friedberg, M. W., Chen, P. G., Van Busum, K. R., Aunon, F. M., Pham, C., \& Caloyeras, J. P. ... Tutty, M. (2013). Factors affecting physician professional satisfaction and their implications for patient care, health systems, and health policy. Santa Monica, CA: American Medical Association, Retrieved from http://www.worldcat.org/title/ factors-affecting-physician-professional-satisfaction-and-their-implications-for-patient-care-health-systems-andhealth-policy/oclc/1008942848

George, M. O. (2010). The lean six sigma guide to doing more with less: cut costs, reduce waste, and lower your overhead. John Wiley \& Sons. Retrieved from https://www.twirpx.com/file/883253/

Hamburg, M. A., \& Collins, F. S. (2010). The Path to Personalized Medicine - Perspective. The New England Journal of Medicine, 363(4), 301-304. doi:10.1056/NEJMp1006304 PMID:20551152

Hsiao, C.-J., Hing, E., \& Ashman, J. (2014). Trends in electronic health record system use among office-based physicians: United States, 2007-2012. National Health Statistics Reports, (75): 1-18. Retrieved from http:// www.ncbi.nlm.nih.gov/pubmed/24844589 PMID:24844589

Jaspers, M. W. M., Smeulers, M., Vermeulen, H., \& Peute, L. W. (2011). Effects of clinical decision-support systems on practitioner performance and patient outcomes: A synthesis of high-quality systematic review findings. Journal of the American Medical Informatics Association, 18(3), 327-334. doi:10.1136/amiajnl-2011-000094 PMID:21422100

Khajouei, R., Peek, N., Wierenga, P. C., Kersten, M. J., \& Jaspers, M. W. M. (2010). Effect of predefined order sets and usability problems on efficiency of computerized medication ordering. International Journal of Medical Informatics, 79(10), 690-698. doi:10.1016/j.jimedinf.2010.08.001 PMID:20833104

Krikunov, A. V., Bolgova, E. V., Krotov, E., Abuhay, T. M., Yakovlev, A. N., \& Kovalchuk, S. V. (2016). Complex data-driven predictive modeling in personalized clinical decision support for Acute Coronary Syndrome episodes. Procedia Computer Science, 80, 518-529. doi:10.1016/j.procs.2016.05.332

Marcilly, R., Ammenwerth, E., Roehrer, E., Niès, J., \& Beuscart-Zéphir, M. C. (2018). Evidence-based usability design principles for medication alerting systems. BMC Medical Informatics and Decision Making, 18(1), 69. doi:10.1186/s12911-018-0615-9 PMID:30041647

Middleton. (2014). Improving Care: Priorities to Improve Electronic Health Record Usability THE AMERICAN MEDICAL ASSOCIATION. Retrieved from https://www.semanticscholar.org/paper/Improving-Care-\%3APriorities-to-Improve-Electronic-Middleton/7dcbc9fc079d09c44a03f1fecb5d5b63a1883a42 
Miller, A., Moon, B., Anders, S., Walden, R., Brown, S., \& Montella, D. (2015). Integrating computerized clinical decision support systems into clinical work: A meta-synthesis of qualitative research. International Journal of Medical Informatics, 84(12), 1009-1018. doi:10.1016/j.ijmedinf.2015.09.005 PMID:26391601

Mohktar, M. S., Lin, K., Redmond, S. J., Basilakis, J., \& Lovell, N. H. (2013). Design of a Decision Support System for a Home Telehealth Application. International Journal of E-Health and Medical Communications, 4(3), 68-79. doi:10.4018/jehmc.2013070105

Payne, T. H., Hines, L. E., Chan, R. C., Hartman, S., Kapusnik-Uner, J., Russ, A. L., \& Malone, D. C. et al. (2015). Recommendations to improve the usability of drug-drug interaction clinical decision support alerts. Journal of the American Medical Informatics Association, 22(6), 1243-1250. doi:10.1093/jamia/ocv011 PMID:25829460

Rose, D., \& Joshi, N. (2018). The Digital Doctor: Hope, Hype, and Harm at the Dawn of Medicine's Computer Age. Annals of Emergency Medicine, 71(1), 154-155. doi:10.1016/j.annemergmed.2017.09.019

Roshanov, P. S., Fernandes, N., Wilczynski, J. M., Hemens, B. J., You, J. J., \& Handler, S. M. ... Haynes, R. B. (2013). Features of effective computerised clinical decision support systems: meta-regression of 162 randomised trials. BMJ (Clinical Research Ed.), 346, f657. doi:10.1136/bmj.f657

Semakova, A., Zvartau, N., Bolgova, E., \& Konradi, A. (2016). Quality of Hypertensive Patients' Electronic Health Records in Specialized Cardiological Centre: 6-Year Trends. In International Conference on Digital Transformation and Global Society (pp. 544-552). Cham: Springer; doi:10.1007/978-3-319-49700-6_54

Sittig, D. F., Wright, A., Osheroff, J. A., Middleton, B., Teich, J. M., Ash, J. S., \& Bates, D. W. et al. (2008). Grand challenges in clinical decision support. Journal of Biomedical Informatics, 41(2), 387-392. doi:10.1016/j. jbi.2007.09.003 PMID:18029232

Slight, S. P., Seger, D. L., Nanji, K. C., Cho, I., Maniam, N., Dykes, P. C., \& Bates, D. W. (2013). Are We Heeding the Warning Signs? Examining Providers' Overrides of Computerized Drug-Drug Interaction Alerts in Primary Care. PLoS One, 8(12), e85071. doi:10.1371/journal.pone.0085071 PMID:24386447

Sobradillo, P., Pozo, F., \& Agustí, Á. (2011). P4 Medicine: the Future Around the Corner. Archivos de Bronconeumología ((English Edition)), 47(1), 35-40. doi:10.1016/S1579-2129(11)70006-4

Sutton, R. S., \& Barto, A. G. (1998). Reinforcement Learning: An Introduction.

Syomov, I. I., Bologva, E. V., Kovalchuk, S. V., Krikunov, A. V., Moiseeva, O. M., \& Simakova, M. A. (2016). Towards Infrastructure for Knowledge-based Decision Support in Clinical Practice. Procedia Computer Science, 100, 907-914. doi:10.1016/j.procs.2016.09.242

Wiser, F., Durst, C., \& Wickramasinghe, N. (2018). Activity Theory: A Comparison of HCI Theories for the Analysis of Healthcare Technology. In Theories to Inform Superior Health Informatics Research and Practice (pp. 235-249). Cham: Springer. doi:10.1007/978-3-319-72287-0_15

Zhang, J., \& Walji, M. F. (2011). TURF: Toward a unified framework of EHR usability. Journal of Biomedical Informatics, 44(6), 1056-1067. doi:10.1016/j.jbi.2011.08.005 PMID:21867774 
Katerina Bolgova is an Associate Professor at High Performance Computing Department, ITMO University. She defended her Ph.D. dissertation in 2012 (thesis subject "Automation of virtual labs development process based on cloud computing") from ITMO University (Saint Petersburg, Russia). Katerina is a part of the team of International Research and Educational Centre of ICT collaborative type - TROIKA (Technology and Research On Information-driven Knowledge Alliance). In 2014, she has become a part of the group of medical data analysis and development of clinical DSS. Her major research interests are complex system simulation, data analysis, research in multidisciplinary areas, and development and internationalization of Russian higher education

Sergey V. Kovalchuk is a senior researcher at eScience Research Institute at ITMO University (Saint-Petersburg, Russia). In 2008 he defended a PhD in computer science on the development of high-performance software system for extreme metocean events simulation. He participates in major R\&D projects of eScience Research Institute, gives lectures and supervises Master students. His current research area covers complex system simulation, highperformance and distributed computing, workflow management systems, knowledge-based eScience technologies.

Marina A. Balakhontceva is a senior lecturer at High-Performance Computing department and a researcher at the eScience Research Institute at ITMO University (Saint-Petersburg Russia). In 2015, she defended her Ph.D. thesis in computer science. Now she manages the project "Methods and technologies for Intelligent integration of heterogeneous data and knowledge sources for decision support in personalized medicine and healthcare" and work on related tasks. Her significant research interests consist of but are not limited to development and implementation of hospital information and decision support systems, medical data analysis, data mining in medicine and healthcare, modeling and simulation of complex systems.

Oleg G. Metsker is a PhD student and an associated researcher at the chair of high-performance computing, ITMO University. His areas of research are complex processes modeling and computational medicine. Conducts applied active research in the field of text mining, data mining, process mining. 